\title{
Der Ruf nach mehr Versorgungsforschung
}

\author{
Call for More Health Services Research
}

Autoren

Institute
Steffi Riedel-Heller ${ }^{1}$, Anke Bramesfeld ${ }^{2}$, Christiane Roick ${ }^{3}$, Thomas Becker ${ }^{4}$, Hans-Helmut König ${ }^{5}$

Universität Leipzig, Klinik und Poliklinik für Psychiatrie, Professur für Public Mental Health

2 Universität Leipzig, Klinik und Poliklinik für Psychiatrie, Forschungsnetz psychische Gesundheit

AOK Bundesverband, Berlin

${ }^{4}$ Universität Ulm, Klinik für Psychiatrie und Psychotherapie II, Günzburg

5 Universität Leipzig, Klinik und Poliklinik für Psychiatrie, Professur für Gesundheitsökonomie
Bibliografie

DOI $10.1055 / \mathrm{s}-2008-1067424$

Psychiat Prax 2008; 35:

157-159

(c) Georg Thieme Verlag KG

Stuttgart · New York .

ISSN 0303-4259

Korrespondenzadresse

Prof. Dr. med.

Steffi G. Riedel-Heller, MPH

Universität Leipzig, Zentrum

für Psychische Gesundheit,

Abteilung Public Health, Klinik und Poliklinik für Psychiatrie Semmelweisstraße 10 04103 Leipzig

Steffi.Riedel-Heller@medizin. uni-leipzig.de

\section{Psychische Störungen - eine wichtige Zielgruppe? \\ $\nabla$}

Psychische Störungen sind häufig und folgenschwer. Die Krankheitslast durch psychische Störungen, gemessen als verlorene Lebensjahre durch Behinderung und Tod, ist enorm. In den westlichen Industrienationen ist sie vergleichbar mit der Krankheitslast, die durch kardiovaskuläre Erkrankungen oder Krebserkrankungen entsteht [1]. Die Relevanz psychischer Störungen, die aus der Bevölkerungsperspektive im direkten Vergleich mit anderen Erkrankungen deutlich wird, wurde lange unterschätzt.

Die Versorgung psychisch Kranker hat sich in den letzten Jahrzehnten dramatisch verändert. Mussten psychisch Kranke bis in die 60er Jahre wohnortfern unter teilweise menschenunwürdigen Bedingungen ihr Dasein in großen Anstalten fristen, wird heute die Mehrzahl psychisch Kranker gemeindenah betreut. Diese mit der Psychiatrieenquete (1975) und analog in den neuen Bundesländern mit den Rodewischer Thesen (1964) eingeleiteten Entwicklungen stimulierten die Versorgungsforschung in der Psychiatrie vergleichsweise früh. Das Angebot gemeindenaher stationärer, ambulanter und komplementärer Dienste zur Versorgung psychisch Kranker ist im bundesweiten Vergleich von einer großen Variabilität geprägt und von einer ausgeprägten Fragmentierung hinsichtlich einer Vielzahl von Leistungserbringern und Leistungsträgern (z. B. Krankenkassen, Rentenversicherungen) gekennzeichnet [2].

\section{Was umfasst Versorgungsforschung? $\nabla$}

Das Spektrum der Versorgungsforschung umfasst Studien zur Epidemiologie und Krankheitslast, zu sozialen und gesundheitsrelevanten Rahmenbedingungen, zu Bedarf, Nachfrage und Inanspruchnahme, zu den ökonomischen Ressour- cen der Versorgung, zu Struktur und Organisation der Versorgung, zum Prozess der Versorgung, sowie zu kurzfristigen Ergebnissen und langfristig zuschreibbaren Resultaten der Versorgung [3]. Das Spektrum reicht somit von den Inputs über die Prozesse zu den Outputs und tatsächlichen Outcomes der Versorgung [4]. Versorgungsforschung kann dabei auf verschiedenen Ebenen angesiedelt sein: der Makro-Ebene der Versorgungssysteme als Rahmenbedingungen, der Meso-Ebene mit ihren einzelnen Versorgungsinstitutionen bzw. ihren institutionellen Arrangements für bestimmte Erkrankungen und der Mikro-Ebene, die sich auf die Analyse einzelner Versorgungs-Interaktionen in unterschiedlichen Behandlungskontexten bezieht.

Das sogenannte „Mission Statement“ der ständigen Kommission Versorgungsforschung (2005) sieht in der Versorgungsforschung einen multidisziplinären Ansatz zur Erforschung der Umsetzung wissenschaftlicher Erkenntnisse in die Praxis der Gesundheitsversorgung hinsichtlich ihrer Wirkung auf Qualität und Effizienz in individueller und soziökonomischer Perspektive [5]. Ein prominentes und deshalb gesondert genanntes Feld methodisch hochwertiger Evaluationsforschung liegt in der Durchführung von Effectiveness-Studien, denen eine Schlüsselrolle für die Anschlussfähigkeit von Versorgungsforschung an die internationale Entwicklung zukommt.

Der Versorgungsforschung lassen sich somit folgende wissenschaftliche Aufgaben zuschreiben: Beschreibung der Versorgungssituation (Beschreibungsfunktion), Analyse der Versorgungssituation (Erklärungsfunktion), Entwicklung von Versorgungskonzepten (Gestaltungsfunktion), wissenschaftliche Begleitung der Implementierung neuer Versorgungskonzepte (Begleitforschungsfunktion) und Evaluation neuer und alter Versorgungskonzepte im medizinischen Alltag (Evaluationsfunktion). 


\section{Boomt die Versorgungsforschung? \\ $\nabla$}

Wer Google bemüht, um nach dem Begriff „Versorgungsforschung“ zu fahnden, wird erstaunt sein: 129000 Treffer bei „auf Deutsch“, 52900 Treffer bei „aus Deutschland“ - kombiniert mit dem Begriff Psychiatrie ergaben sich analog 11200 und 10400 Treffer. Trotzdem führt die Versorgungsforschung neben grundlagenorientierter und krankheitsorientierter Forschung in der deutschen medizinischen Forschungslandschaft noch immer eine Randexistenz [6], von einem Boom kann keine Rede sein. Aber es ist zumindest einiges in Bewegung gekommen, spätestens seit der Sachverständigenrat für die Konzertierte Aktion im Gesundheitswesen in seinem Gutachten von 2001/2002 den „international betrachtet erheblichen Nachholbedarf“ einer intensiven Versorgungsforschung anmahnte [7]. Meilensteine in der bisherigen Entwicklung der Versorgungsforschung waren die Etablierung eines sechsjährigen Forschungsprogramms „Versorgungsforschung“ durch das Bundesministerium für Bildung und Forschung und die Spitzenverbände der Gesetzlichen Krankenversicherungen im Jahr 1999, und daran anknüpfend, das 2005 auf dem deutschen Ärztetag beschlossene Programm „Versorgungsforschung“ der Bundesärztekammer sowie die Veröffentlichung zweier Memoranden zur Versorgungsforschung 2003 und 2005 durch die Ständige Kongresskommission des Deutschen Kongresses für Versorgungsforschung. Sogar die Deutsche Forschungsgemeinschaft, die in der Vergangenheit die Versorgungsforschung nicht in wesentlichem Umfang gefördert hatte, will sich mehr auf diesen Bereich einlassen [8].

\section{Forschung zur Versorgung psychisch Kranker - was könnten zentrale Themen sein? \\ $\nabla$}

Die Versorgung psychisch Kranker - in all ihren Facetten - ist ein weites Feld. Welche Themen drängen besonders? Am 22.04.2008 haben sich in Berlin Wissenschaftler und Akteure aus dem Feld getroffen, um Themen der Versorgungsforschung im Bereich psychische Gesundheit zu diskutieren, die Ihnen unter den Nägeln brennen. Eine erste Zusammenschau ergab eine Reihe von Arbeitsfeldern, die in einem weiteren Prozess (DelphiTechnik) priorisiert werden sollen.

Einige seien hier genannt: Epidemiologische Daten liefern wertvolle Hinweise zur Unter- und Fehlversorgung psychischer Störungen. Hier ist es sinnvoll, nicht nur auf Surveydaten zurückzugreifen, sondern auch mit Routinedaten zu arbeiten. Barrieren für die Inanspruchnahme von Versorgungsleistungen (auf Seiten der Betroffenen, der Helfer und des Systems) bedürfen einer genauen Analyse. Ein Forschungsdefizit wurde im ambulanten und komplementären Bereich unter besonderer Berücksichtigung von Schnittstellen zu anderen Hilfesystemen ausgemacht. Wichtig erscheint auch die systematische Integration von Selbstmanagementelementen bei der Behandlung psychisch Kranker voranzutreiben. Versorgungsforschung soll Aufschlüsse über Ursachen und Konsequenzen der großen regionalen Variabilität der Versorgung geben und ein Benchmarking fördern. Zudem wird Forschungsbedarf im Bereich der ambulanten Rehabilitation psychisch Kranker unter besonderer Berücksichtigung innovativer Ansätze in der Arbeitsrehabilitation psychisch Kranker gesehen. Neue Finanzierungsmodelle psychiatrischer Versorgung (regionales Budget, persönliches Budget) stehen weiter im Zentrum des Interesses. Die gesundheitsökonomische Perspektive ist integraler Bestandteil von Versorgungsforschung, um Wirk- samkeit und Kosteneffektivität von Gesundheitsleistungen zu erfassen. Der Einbezug der Nutzer- und Angehörigenperspektive ist unerlässlich. Bürger mit Migrationshintergrund und alte Menschen sind eine besonders wichtige Zielgruppe für die Versorgungsforschung. Der Einfluss der Komorbidität von körperlichen und psychischen Störungen auf die Versorgung soll zunehmend Berücksichtigung finden.

\section{Die Psychiatrische Praxis ein Forum fiir Versorgungsforschung?} $\nabla$

Die Psychiatrische Praxis hat die Entwicklung der psychiatrischen Versorgung wissenschaftlich begleitet [9]. Einer der ersten Schwerpunkte, der bis heute hohes Gewicht hat, war die Konzeptentwicklung psychiatrischer Dienste sowie deren Umsetzung und Evaluation [10,11]. So konnte auch die Hälfte (49\%) der Originalarbeiten der Jahre 2006 und 2007 der Versorgungsforschung zugeordnet werden. Die große Mehrheit dieser Arbeiten beschäftigt sich mit der Beschreibung und Analyse der Versorgungssituation sowie mit der Evaluation von Versorgungskonzepten im Alltag. Der ambulante und komplementäre Bereich psychiatrischer Versorgung bleibt nach wie vor unterbelichtet [10].

Obgleich die Bedeutung ökonomischer Aspekte bei der Evaluation psychiatrischer und psychotherapeutischer Versorgung außer Zweifel steht [12], besteht nach wie vor ein Defizit an gesundheitsökonomischer Evidenz im Bereich der Versorgung psychisch Kranker $[13,14]$. Dies zeigt sich auch in der Psychiatrischen Praxis: Nur 10\% der 2006 bzw. 2007 veröffentlichten Arbeiten untersuchten eine gesundheitsökonomische Fragestellung. In vier Studien wurden die Kosten von ambulanter, tagesklinischer bzw. vollstationärer Behandlung von psychiatrischen Patienten erfasst und zum Teil verglichen [15-18]. Eine Studie berichtete Ergebnisse zur Krankheitslast von Depressionen [19]. Weiterführende gesundheitsökonomische Analysen, sogenannte Effizienzstudien, wurden nicht vorgelegt.

Die Psychiatrische Praxis versteht sich traditionell als das Forum für die Versorgungsforschung im psychosozialen Bereich und will damit zu einer bisher unzureichend etablierten Diskussionskultur beitragen, die es erlaubt, sich mit Ergebnissen der Versorgungsforschung durchaus kontrovers, jedoch nicht ideologisch-interessengeleitet auseinanderzusetzen [5].

\section{Literatur}

1 Lopez AD et al. Global and regional burden of disease and risk factors, 2001. Lancet 2006; 367: 1747-1757

2 Gesundheitsministerkonferenz der Länder 2007 (AG Psychiatrie der Obersten Landesgesundheitsbehörden) (Hrsg). Psychiatrie in Deutschland - Strukturen, Leistungen, Perspektiven. Baden Württemberg: 2007; Auflage 1 http://www.gmkonline.de/_beschluesse/Protokoll_ 80-GMK_Top1002_Anlage1_Psychiatrie-Bericht.pdf

3 Schwartz FW, Badura B, Busse R, Leidl R, Raspe H, Siegrist J (Hrsg). Das Public Health Buch: Gesundheit und Gesundheitswesen. München, Jena: Urban \& Fischer Verlag, 2003

4 Pfaff $H$. Versorgungsforschung - Begriffsbestimmung, Gegenstand, Aufgaben. In: Pfaff H, Schrappe M, Lauterbach KW, Engelmann U, Halber M (Hrsg): Gesundheitsversorgung und Disease Management. Bern: Hans Huber, 2003: 13-23

5 Schrappe M, Glaeske G, Gottwik M, Kilian R, Papadimitriou K, ScheidtNave C, Schulz KD, Ziegenhagen D, Pfaff H. Memorandum II zur Versorgungsforschung in Deutschland: Konzeptionelle, methodische und strukturelle Voraussetzungen der Versorgungsforschung; 2005 www.dkvf.de/content/e36/e174/MemorandumII.pdf (13.02.2008) 
6 Der Status quo der Förderung von Versorgungsforschung in Deutschland. Forschungs-Förderung dringend gesucht. Monitor Versorgungsforschung 2008; $1: 11$ - 12

7 SVR (Sachverständigenrat für die Konzertierte Aktion im Gesundheitswesen). Bedarfsgerechtigkeit und Wirtschaftlichkeit. Bände III.1 - III.3: Über-, Unter- und Fehlversorgung. Baden-Baden: 2002

8 Gerst T. Versorgungsforschung: Was überhaupt, wozu und womit? Dtsch Arztebl 2006; 103 (12): A-752/B-639/C-619

9 Finzen A. Psychiatrische Praxis: Das dreißigste Jahr. Psychiat Prax 2003; 30: 413

10 Claassen D, Priebe S. Deutschsprachige psychiatrische Versorgungsforschung - Was und wie wird berichtet? Psychiat Prax 2003; 30: 414 423

11 Holzinger A, Angermeyer MC. Sozialpsychiatrische Forschung im deutschen Sprachraum. Psychiat Prax 2002; 29: $397-410$

12 Kilian R, Becker T. Aktuelle Entwicklungstendenzen psychiatrischer Evaluationsforschung. In: Pawils S, Koch U (Hrsg): Psychosoziale Versorgung in der Medizin. Stuttgart: Schattauer Verlag, 2006: 331 - 342

13 Salize HJ. Gesundheitsökonomische Untersuchungen in der Versorgung psychisch Kranker. Die Psychiatrie 2005; 2: 165-172
14 König HH, Friemel S. Gesundheitsökonomie psychischer Krankheiten. Bundesgesundheitsblatt 2006; 49 (1): 46 - 56

15 Haberfellner EM, Grausgruber A, Grausgruber-Berner R, Ortmair M, Schöny W. Die Enthospitalisierung ehemaliger psychiatrischer Langzeitpatienten in Oberösterreich - Inanspruchnahme von Versorgungsleistungen und Kosten der außerstationären Versorgung. Psychiat Prax 2006; 33: 74-80

16 Weithmann G, Hoffmann M. Häufigkeit und Kosten zusätzlicher stationärer Behandlungen im Jahr nach tagesklinischer oder vollstationärer Alkoholentzugsbehandlung. Psychiat Prax 2007; 34: 15 - 19

17 Stamm K, Merkel S, Mann K, Salize HJ. Welche Kosten verursachen alkoholkranke Versicherte? - Eine Analyse aus Sicht einer Betriebskrankenkasse. Psychiat Prax 2007; 34: 194-199

18 Kallert TW, Schönherr R, Frohling D, Schützwohl M. Patientenbezogene Therapiekosten akutpsychiatrischer tagesklinischer und vollstationärer Behandlung. Psychiat Prax 2007; 34: 377-383

19 Günther OH, Friemel S, Bernert S, Matschinger H, Angermeyer MC, König HH. Die Krankheitslast von depressiven Erkrankungen in Deutschland. Psychiat Prax 2007; 34: 292-301

\section{Nachruf auf Prof. Dr. Hans Klaus Rose}

$\nabla$

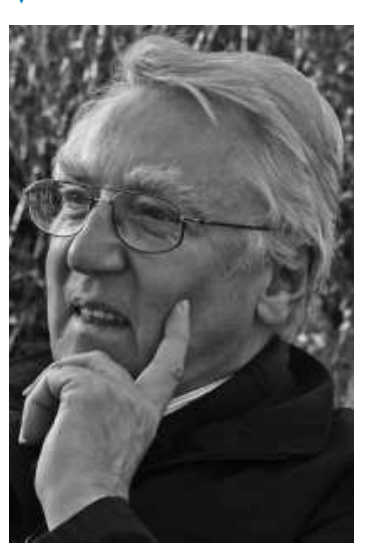

Prof. Dr. Hans Klaus Rose ist tot. Er starb am 9.4.2008, 75-jährig, in Hannover.

Dorthin hatte ihn 1967 Karl Peter Kisker von Heidelberg aus als Oberarzt mitgenommen, und in dieser Funktion blieb er zunächst viele Jahre, bis er selbstständiger Leiter der neu geschaffenen gerontopsychiatrischen Abteilung an der Medizinischen Hochschule Hannover wurde. 1987 wurde er zum Ärztlichen Direktor der Stiftung Tannenhof (Remscheid) berufen. Für die dort geleistete Arbeit erhielt HKR, wie wir Hannoveraner ihn nannten, bei seinem Abschied das Bundesverdienstkreuz.

Nach seiner Pensionierung im Jahre 1998 zog er mit seiner Familie wieder nach Hannover zurück, betrieb dort noch einige Jahre lang eine kleine Privatpraxis, war forensisch tätig und kümmerte sich um seine Enkelkinder.

Hans Klaus Rose war, zusammen mit Helmut Köster (Düren), Asmus Finzen (damals Wunstorf) und ganz am Anfang auch Manfred Bauer (damals Hannover), Mitbegründer der von K. P. Kisker ins Leben gerufenen Zeitschrift „Psychiatrische Praxis“, die er bis zum Jahre 1993 federführend und souverän leitete. Ihre Zukunft lag ihm bis zuletzt ausgesprochen am Herzen.

Seine frühen sozialpsychiatrischen Publikationen waren für uns Jüngere damals wichtige Grundlagentexte, die bis heute von ihrer Bedeutung wenig verloren haben. Einer davon „Grundfragen der Teamarbeit in der Psychiatrie“ ist in den Sammelband „Sozialpsychiatrie vor der Enquete“ (Psychiatrie-Verlag, 1997) aufgenommen worden.

Mit dem Tod von Hans-Klaus Rose verliert die deutsche Psychiatrie einen aufrechten, zugleich kritischen Sozialpsychiater der ersten Stunde, der sich zeit seines Lebens von modischen Strömungen ebensowenig beeindrucken ließ wie von purem Aktionismus. Das sei ihm gedankt.

Begonnen hatte alles in der kleinen Anhaltiner Stadt Zerbst. Dort war sein Vater chirurgischer Chefarzt am Kreiskrankenhaus. Er wurde 1948 von den neuen Machthabern aus dem Amt vertrieben und steckbrieflich gesucht. In letzter Minute floh er mit seiner Frau, seinen beiden Söhnen und der Tochter nach Landau in der Pfalz, wo seine Schwägerin lebte. Im nahe gelegenen Neustadt fand er in dem, im Entstehen begriffenen, Vertauensärztlichen Dienst eine Stelle und hatte diesen Posten bis zu seiner Pensionierung inne.

Hans Klaus Rose hat gelegentlich im Freundeskreis darüber gesprochen, dass diese Flucht, so schlimm sie für die Familie insgesamt war, für ihn viel Gutes mit sich brachte. Er konnte, wie auch sein allzu früh verstorbener Bruder, nach dem auf einem humanistischen Gymnasium abgelegten Abitur 1954 Medizin studieren, bestand 1959 in Heidelberg sein Staatsexamen und promovierte im gleichen Jahr bei dem Physiologen Schäfer, mit einer experimentellen Arbeit über Bluthochdruck bei Ratten. Zeitgleich hatte er ein, von der „Studienstiftung“ gefördertes Philosophiestudium aufgenommen, das ihm beim späteren Denken und Schreiben, nicht zuletzt aber auch im psychiatrischen Alltag, ausgesprochen hilfreich war.

Nach dem Ende der damals noch zweijährigen Medizinalassistentenzeit verschrieb er sich der Psychiatrie und blieb bei diesem Fach, das wie kein anderes die Natur- und die Geisteswissenschaften miteinander verbindet.

Noch auf der Schule hatte er seine spätere Frau Gisela, eine Klassenkameradin, kennen und lieben gelernt und sie 1960 geheiratet. Die Kinder ließen nicht lange auf sich warten. 1962 wurde Bettina geboren, 1963 Christoph und 1968 Natali. Die ersten beiden wurden wie ihr Vater und ihr Großvater Ärzte, der Nachkömmling war eher künstlerisch begabt und blieb bis heute in diesem Metier.

Die Welt der Roses schien in jeder Hinsicht in Ordnung. Bis zu Bettinas Tod im Jahre 2002. Wenn Kinder vor ihren Eltern sterben, ist dies für alle Beteiligten eine Katastrophe für deren Beschreibung die Worte fehlen.

Der Psychiater Hans Klaus Rose hinterlässt eine Frau, zwei Kinder und fünf Enkelkinder.

Manfred Bauer, Offenbach

Mark Richartz, Maastricht 